\title{
Editorial
}

Theme: Recent Trends in the Development of Chitosan-Based Drug Delivery Systems

Guest Editors: Claudio Salomon, Francisco Goycoolea, and Bruno Moerschbacher

\section{Recent Trends in the Development of Chitosan-Based Drug Delivery Systems}

\author{
Claudio Salomon, ${ }^{1,4}$ Francisco M. Goycoolea, ${ }^{2}$ and Bruno Moerschbacher ${ }^{3}$
}

Received 14 March 2017; accepted 14 March 2017; published online 28 March 2017

KEY WORDS: chitosan; chitin; biopolymer; drug delivery systems.

The 2015 12th International Conference of the European Chitin Society jointly with 13th International Conference on Chitin and Chitosan (EUCHIS/ICCC 2015) was held from August 30th to September 2nd in Münster, Germany. This was the first ICCC held in Germany and the first one to be cosponsored by AAPS and AAPS PharmSciTech journal, the International Union of Pure and Applied Chemistry (IUPAC), the European Chitin Society (EUCHIS), the University of Münster, along with several companies.

With more than 300 attendees from all over the world, it was also probably the largest ever chitin and chitosan conference and the first one with a sizeable industrial exhibition. A Young Researcher Symposium preceded the conference, and it was followed by three parallel postconference workshops, namely on Chitosan Analysis, Mucoadhesion, and Electrospinning, the latter sponsored by the COST Action MP1206.

The AAPS PharmSciTech journal sponsored the Award to the Best Poster in the Pharmaceutical Sciences. The poster prize was awarded to Ayben Işılay Doğan, Gulcin Akça, and Sevda Şenel from Hacettepe University (Turkey) for their work on Chitosan based formulations of atorvastatin for periodontal delivery.

Chitosan has long been recognized as one of the most promising functional biopolymers, with potential applications in areas as diverse as material, food, agricultural, biomedical, and pharmaceutical sciences. Research of the past decade or

\footnotetext{
$\overline{{ }^{1} \text { Facultad de Cs. Bioquímicas y Farmacéuticas, Universidad Nacional }}$ de Rosario, IQUIR-CONICET, Suipacha 531, 2000, Rosario, Argentina.

${ }^{2}$ School of Food Science and Nutrition, University of Leeds, Leeds, LS2 9JT, UK.

${ }^{3}$ Institut für Biologie und Biotechnologie der Pflanzen Westfälische Wilhelms-Universtät Münster, Schlossgarten 3, 48149, Münster, Germany.

${ }^{4}$ To whom correspondence should be addressed. (e-mail: csalomon@fbioyf.unr.edu.ar)
}

so has paved the way for today's "second generation" chitosans with better-defined structures and more reliable functions than the early ill-defined "first-generation" materials.

This special volume of AAPS PharmSciTech, entitled "Theme: Recent Trends in the Development of ChitosanBased Drug Delivery Systems," includes a topical collection of a total of 14 papers including both articles from EUCHIS/ ICCC 2015 plus others freely contributed manuscripts that successfully passed the normal rigorous peer review process addressing a diversity of topics.

The first article describes the advantages of chitosan matrices for the development of buccal mucoadhesive devices. These types of biopolymeric formulations generate hydrophilic networks that contain numerous polar functional groups that interact with biological structures. Tejada et al. [1] describe the formulation of miconazole nitrate buccal films using chitosan, pectin, and hydroxypropylmethyl cellulose to form such polymeric matrices. Chitosan:hydroxypropylmethyl cellulose films were found to be the most appropriate formulations in terms of folding endurance, mechanical properties, and adhesiveness. The in vitro antifungal activity showed a significant activity of the model drug loaded into those films.

Reduction of particle size is an attractive and widely used technique to improve the aqueous solubility of poorly watersoluble compounds belonging to the class II of the Biopharmaceutical Classification System. Herein, Priotti et al. [2] reported the preparation of albendazole microcrystals using the bottom-up technology based on chitosan and cellulose derivatives. The concentration of polymers, the influence of surfactant in the crystals, the variables area under the curve, albendazole microcrystal solubility, and drug released were analyzed with a three-way ANOVA. The in vitro studies demonstrated the suitability of this approach for further in vivo studies.

Microencapsulation is still one of the most suitable procedures to prepare controlled drug delivery systems, and the type of selected polymers used as carrier is one of the key 
factors in this issue. Herein, Khattab et al. [3] explored the delivery of ranitine $\mathrm{HCl}$ from mucoadhesive polymeric microspheres using chitosan and two cellulose derivatives as wall materials. A $3 \times 22$ full factorial design was applied to find the optimized formulation in terms of particle size, swelling index, drug entrapment efficiency, and bioadhesion drug release. In vivo protective and healing effects of the optimized formula against gastric ulcers were investigated, also.

Chitosan and its synthetic derivatives have been used as biomaterial in several fields including tissue engineering due to, mainly, its good biocompatibility and biodegradability. In this issue, an interesting application of chitosan derivatives has been described by Martínez-Campos et al. [4]. Three types of chitosan-based films were prepared and evaluated, in terms of cell adhesion and proliferation, using pre-myoblastic cells and B16 cells as a tumoral model. A differential cell behavior was detected on chitosan films due to their different surface modification. B16 cells have shown lower vinculin expression when cultured on sulfonated chitosan films.

In the last decades, different nanotechnologies have been employed to improve the technological and biopharmaceutical performance of hydrophobic drugs through the formulation of nano-drug delivery systems. In particular, drug nanosuspensions emerged as an attractive approach to deliver poorly water-soluble molecules. Kassem et al. [5] described the preparation of nanosuspensions of lacidipine, a highly lipophilic calcium channel blocker. A Box-Behnken design was used to optimize the formulation variables. A significantly improved dissolution rate due to particle size reduction and decreased crystallinity was obtained, suggesting that this novel alternative could be a valuable tool to improve oral absorption of lacidipine.

Another application of chitosan in the nanotechnology field has been described by Zhao et al. [6]. Timolol maleate, a compound to treat high pressure inside the eye due to glaucoma, was incorporated into chitosan nanoparticles. Nano-formulations were prepared and optimized via a fourlevel and three-factor Box-Behnken statistical experimental design. In vitro transcorneal permeation study, confocal microscopy, and in vivo pharmacodynamic were performed, and the results indicated the suitability of the methodology for the treatment of ocular diseases.

As already mentioned, polymeric mucoadhesion is an interesting and valuable property in the design of drug delivery systems. In this regard, chitosan is able to form electrostatic complexes under acidic medium with the anionic groups of the mucus gel layer. As a consequence, an extended residence time of the mucoadhesive polymeric device at the site of drug absorption can be observed. Herein, Alkhader et al. [7] investigated the properties of a mucoadhesive chitosan-pectinate nano-formulation for colonic delivery of curcumin. A detailed physicochemical characterization was carried out and it was found that $80 \%$ release of curcumin was achieved in pectinase-enriched medium.

In recent years, chitosan and its derivatives have gained increased importance for wound healing, which consist of different steps to repair the damage of tissue and replacement of lost skin. Aslan et al. [8] described the formulation of interleukin-2-loaded chitosan-based nanogels for the healing of wound incision in rats. Artificial neural network models were developed using selected input parameters where particle size was an output parameter for interleukin-2-free nanogels. Wound healing effect of these nanogels was evaluated by determining the malondialdehyde (MDA) and glutathione (GSH) levels of wound tissues in rats. Interleukin-2 was released slowly after the initial burst effect and in vivo study showed that this approach was effective for improving wound healing in rats.

Regulation of gene expression is generally described as gene silencing and it is related with the cell ability to prevent the expression of a certain gene. One of the most useful approach to avoid the gene expression is by means of by small interfering RNA (siRNA). Herein, Salva et al. [9] deeply investigated the in vitro gene expression of chitosanbased siRNA nanoplexes to inhibit cell proliferation in proliferative glomerulonephritis disease. After transfection, chitosan nanoplexes successfully diminished cell proliferation and cell migration in the rat mesangial cells by inhibiting the grow factors suggesting that the formulation of chitosanbased gene delivery system is a promising tool to treat renal disease.

Another work based on the mucoadhesion of chitosan is herein reported by Naz et al. [10]. Unmodified chitosan was covalently modified by thioglycolic acid (TGA) and cysteine to design multifunctional thiolated polymeric films for the delivery of fluconazole, an anfigungal agent. The thiolated mucoadhesive films were characterized for water-uptake capacity, mucoadhesion, permeability study, and in vitro drug release profile. Through the formulation of these thiopolymeric devices, both permeation and film mucoadhesion were highly increased while a controlled fluconazole release was obtained compared to the unmodified formulation.

Next, Mostafa et al. [11] described another application of chitosan as biomaterial carrier by formulating chitosannanobioactive glass scaffolds loaded with ciprofloxacin for bone tissue engineering. The morphology of the matrices was fully characterized indicating that the chitosan-bioactive glass formulation (1:2 ratio) was the most suitable scaffold composition for controlled drug release. The authors also confirmed that the optimized polymeric matrix showed a significant biocompatibility by inducing cell growth and differentiation.

The efficient and safe protein delivery from biopolymeric devices is an emerging field in pharmaceutical research; however, several challenges need to be resolved including the interactions between the carrier and the protein and its release from the polymeric network. In this context, Becerra et al. [12] reported the preparation of chitosan-based films to deliver ovalbumin, as model protein. A systematic physicochemical study was performed to evaluate whether the polymeric degree of acetylation would influence both the film crystalline structure and its release characteristics. The authors confirmed that hydrophilic/hydrophobic interactions related with the chitosan degree of acetylation exhibit a significant influence over the matrix swelling behavior and further release of the model protein.

The formulation of sustained-release dosage forms involves the formation of crosslinked polymeric networks, which may avoid a high and not desirable burst release. However, more information to find the suitable formulation in terms of production procedure, size distribution, type of 
crosslinking agent, and drug release rate is still required. In this issue, Rodriguez et al. [13] reported the preparation of chitosan microparticles crosslinked with different ratios of sodium tripolyphosphate microparticles. The impact of the agitation rate, oil phase concentration, emulsifier to oil ratio, and polymer concentration over the loading and further release of acetylsalicylic acid, a model drug, was analyzed in detail. The authors reported that size regulation of aqueous droplets in the oil phase was achieved by using a right balance among the components of the formulation.

Chitosan is also widely used as starting material for the preparation of microbeads. An original research by Xing et al. [14] reported the successful encapsulation of specific egg yolk immunoglobulin (IgY) to avoid the binding of clostridium difficile toxins to the colonic mucosa. The authors confirmed that the chitosan-Ca pectinate microbead formulations exhibited promising colon-targeting properties, showing low drug release at both the stomach and small intestine while a fast release was observed in colon. It was concluded that these chitosan-based microbeads would represent a convenient approach for specific immunoglobulin release into the colon.

The advantages of pulmonary nano-drug delivery systems are mainly based on a better effectiveness to the target site, improved drug efficacy, and increased bioavailability. Herein, Komenek et al. [15] designed gold-based nanoparticles stabilized by quaternized chitosan-gallic acid-folic acid for the treatment of lung cancer. In vitro cytotoxicity studies, cellular uptake, and localization of such nanocarrier were deeply described by the authors. As a conclusion, they found that this gold-based nanocarrier may enhance the cellular uptake and reduce the accumulation of nanoparticles in the pulmonary tract leading to less toxicity profile than other nanocarriers.

\section{REFERENCES}

1. Tejada G, et al. Development and evaluation of buccal films based on chitosan for the potential treatment of oral candidiasis. AAPS PharmSciTech. 2017; doi:10.1208/s12249-017-0720-6.

2. Priotti J, et al. Albendazole microcrystal formulations based on chitosan and cellulose derivatives: physicochemical characterization and in vitro parasiticidal activity in Trichinella spiralis adult worms. AAPS PharmSciTech. 2016; doi:10.1208/s12249016-0659-z.

3. Khattab A, et al. Optimization and evaluation of gastroretentive ranitidine $\mathrm{HCl}$ microspheres by using factorial design with improved bioavailability and mucosal integrity in ulcer model. AAPS PharmSciTech. 2017; doi:10.1208/s12249-017-0744-y.

4. Martínez-Campos E, et al. Cell adhesion and proliferation on sulfonated and non-modified chitosan films. AAPS PharmSciTech. 2016; doi:10.1208/s12249-016-0619-7.

5. Kassem M, et al. Enhanced solubility and dissolution rate of lacidipine nanosuspension: formulation via antisolvent sonoprecipitation technique and optimization using BoxBehnken design. AAPS PharmSciTech. 2016; doi:10.1208/ s12249-016-0604-1.

6. Zhao R, et al. Development of timolol-loaded galactosylated chitosan nanoparticles and evaluation of their potential for ocular drug delivery. AAPS PharmSciTech. 2017; doi:10.1208/ s12249-016-0669-x.

7. Alkhader E, et al. Mucoadhesive chitosan-pectinate nanoparticles for the delivery of curcumin to the colon. AAPS PharmSciTech. 2016; doi:10.1208/s12249-016-0623-y.

8. Aslan C, et al. Development of interleukin-2 loaded chitosanbased nanogels using artificial neural networks and investigating the effects on wound healing in rats. AAPS PharmSciTech. 2016; doi:10.1208/s12249-016-0662-4.

9. Salva E, et al. Inhibition of glomerular mesangial cell proliferation by siPDGF-B- and siPDGFR- $\beta$-containing chitosan nanoplexes. AAPS PharmSciTech. 2016; doi:10.1208/s12249016-0687-8.

10. Naz K, et al. Formulation and in vitro characterization of thiolated buccoadhesive film of fluconazole. AAPS PharmSciTech. 2016; doi:10.1208/s12249-016-0607-y.

11. Mostafa AA, et al. Bioactive/natural polymeric scaffolds loaded with ciprofloxacin for treatment of osteomyelitis. AAPS PharmSciTech. 2016; doi:10.1208/s12249-016-0605-0.

12. Becerra J, et al. Tuning the hydrophilic/hydrophobic balance to control the structure of chitosan films and their protein release behavior. AAPS PharmSciTech. 2016; doi:10.1208/s12249-0160678-9.

13. Rodriguez LB, et al. Effect of formulation and process parameters on chitosan microparticles prepared by an emulsion crosslinking technique. AAPS PharmSciTech. 2016; doi:10.1208/ s12249-016-0677-x.

14. Xing P, et al. Colon-targeted delivery of IgY against clostridium difficile toxin A and B by encapsulation in chitosan-Ca pectinate microbeads. AAPS PharmSciTech. 2016; doi:10.1208/s12249016-0656-2.

15. Komenek S, et al. Nanogold-gallate chitosan-targeted pulmonary delivery for treatment of lung cancer. AAPS PharmSciTech. 2016; doi:10.1208/s12249-016-0644-6. 